\title{
First deep underground observation of rotational signals from an earthquake at teleseismic distance using a large ring laser gyroscope
}

\author{
$\underline{\text { Andreino Simonelli }}{ }^{1,3,6, *}$, Jacopo Belfi ${ }^{1}$, Nicolò Beverini ${ }^{1,2}$, Giorgio Carelli ${ }^{1,2}$, Angela Di Virgilio ${ }^{1}$, \\ Enrico Maccioni ${ }^{1,2}$, Gaetano De Luca ${ }^{5}$, Gilberto Saccorotti ${ }^{4}$ \\ *andreino.simonelli@pi.infn.it \\ ${ }^{1}$ Istituto Nazionale di Fisica Nucleare, Pisa, Italy \\ ${ }^{2}$ Università di Pisa, Dipartimento di Fisica "E. Fermi", Pisa, Italy \\ ${ }^{3}$ Ludwig-Maximilians-Universität, Munich, Germany \\ ${ }^{4}$ Istituto Nazionale di Geofisica e Vulcanologia, Pisa, Italy \\ ${ }^{5}$ Istituto Nazionale di Geofisica e Vulcanologia, Centro Nazionale Terremoti, Rome, Italy
}

\begin{abstract}
Recent advances in large ring laser gyroscopes (RLG) technologies opened the possibility to observe rotations of the ground with sensitivities up to $10^{-11} \mathrm{rad} / \mathrm{sec}$ over the frequency band of seismological interest $(0.01-1 \mathrm{~Hz})$, thus opening the way to a new geophysical discipline, i.e. rotational seismology. A measure of rotations in seismology is of fundamental interest for (a) the determination of all the six degrees of freedom that characterize a rigid body's motion, and $(b)$ the quantitative estimate of the rotational motions contaminating ground translation measurements obtained from standard seismometers. Within this framework, this paper presents and describes GINGERino, a new large observatory-class RLG located in Gran Sasso underground laboratory (LNGS), one national laboratories of the INFN (Istituto Nazionale di Fisica Nucleare). We also report unprecedented observations and analyses of the roto-translational signals from a tele-seismic event observed in such a deep underground environment.
\end{abstract}

\section{INTRODUCTION}

Thanks to the optical measurement principle, Ring Laser Gyroscopes (RLG) are insensitive to translations, thus representing the best sensors for capturing the tiny rotational motions associated with the transit of seismic waves. The need for a rotational sensor in geophysics was first claimed in a fundamental text about seismology (Aki and Richards (2009)), where the authors state that "... note the utility of measuring rotation near a rupturing fault plane (...), but as of this writing, seismology still awaits a suitable instrument for making such measurements". The search for such a sensor is of actual interest, as shown by many recent studies (Kaláb et al. (2013); Brokešová and Málek

"'Regional doctoral school in Earth Sciences", XXX cycle, Pisa, Italy
(2010); Schreiber et al. (2006a)). So far, RLGs allowed to achieve important results in a variety of disciplines, spanning from geodesy (Schreiber et al. (2004)) to both regional and global seismology (Igel et al. (2005); Pancha et al. (2000); Simonelli (2014); Schreiber et al. (2006a)). Depending on the scope, the size or RLG changes from some centimeters to more than four meters. RLGs for navigation are very small and lightweight; they are produced commercially and are widely adopted on either underwater or airborne platforms. Their sensitivity, however, is not sufficient for geophysical applications. Sensitivity and accuracy of RLGs increase with size: maximizing the dimensions of the apparatus implies a minimization of those physical effects that provoke departure from an ideal linear regime. Scientific results like monitoring of the solid-Earth tides or a 
measure of the length of the day (LOD) are only achievable by very-large-frame RLGs. At present, the G-ring deployment in Wettzel (Germany) represents the reference RLG for geodetic and seismological observations. Smaller in size and less expensive are RLGs of the class Geosensor, (Schreiber et al. (2006b); Belfi et al. $(2012 b, a))$. Recently, the Italian National Institute for Nuclear Physics (INFN hereinafter) supported the development of the GINGERino RLG. Within the context of a larger project of fundamental physics, this apparatus is intended to reach the high sen- sitivity needed to observe general relativity effects, by the same time providing crucial data for geophysical and seismological applications (for more detail see the URL https://web2.infn.it/GINGER/ index.php/it/).

\section{INSTRUMENTAL APPARATUS}

The GINGERino is located within the INFN's Gran Sasso National Laboratory (LNGS) (Fig. $1)$.

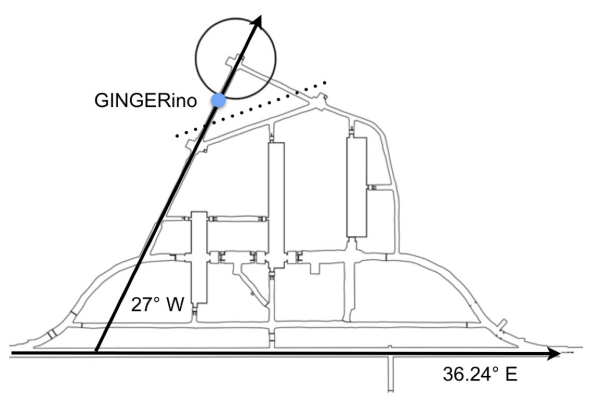

Figure 1: Map of the LNGS underground laboratories

The equipment of geophysical and seismological interest is constituted by the following instruments: The large He:Ne ring laser visible in Fig. 2; this is a $3.6 \mathrm{~m}$ side square cavity ring laser installed over a granite structure block anchored to the rock of the B knot tunnel of the LNGS. This is our rotation sensor, it is able to detect rotations around the symmetry axis (oriented vertically) with a sensitivity better than $10^{-10} \mathrm{rad} / \mathrm{s}$ in the band of interest for global seismology (5Hz-300s). A Nanometrics Trillium 240s seismometer installed at the center of the RLG granite frame Fig. 3, providing the ground translation data to be compared to the RLG rotational measurements for shear and surface waves from either micro-seismic or earthquakes sources.

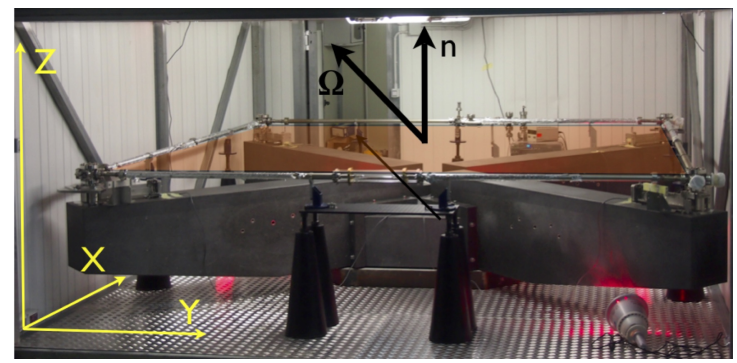

Figure 2: The GINGERino RLG

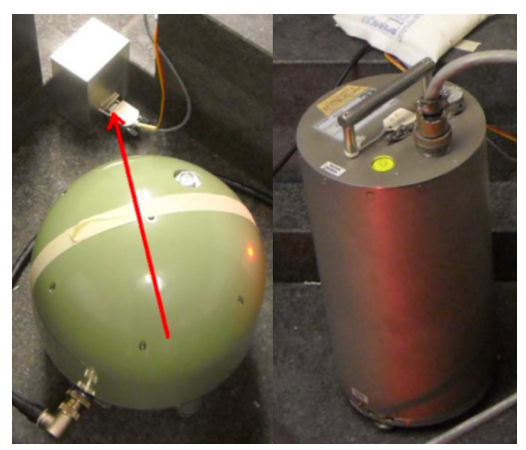

Figure 3: The NANOMETRICS Trillium $240 \mathrm{~s}$ (left) and Guralp CMG 3T360s (right) and the Lippmann 2-K tilt meter (on top), the red arrow shows the North direction

This instrument is also part of the national earthquake monitoring program of the Istituto Nazionale di Geofisica e Vulcanologia (INGV hereinafter; see also the URL http://iside.rm.ingv.it/iside/standard/ info_stazione.jsp?page=sta\&sta $=2571$. A second broadband seismometer, Guralp CMG 3T-360s (Fig. 3) is placed in the central block for data redundancy. A Lippmann 2-K digital tilt-meter with a resolution better then one nrad is placed beside the seismometer in 

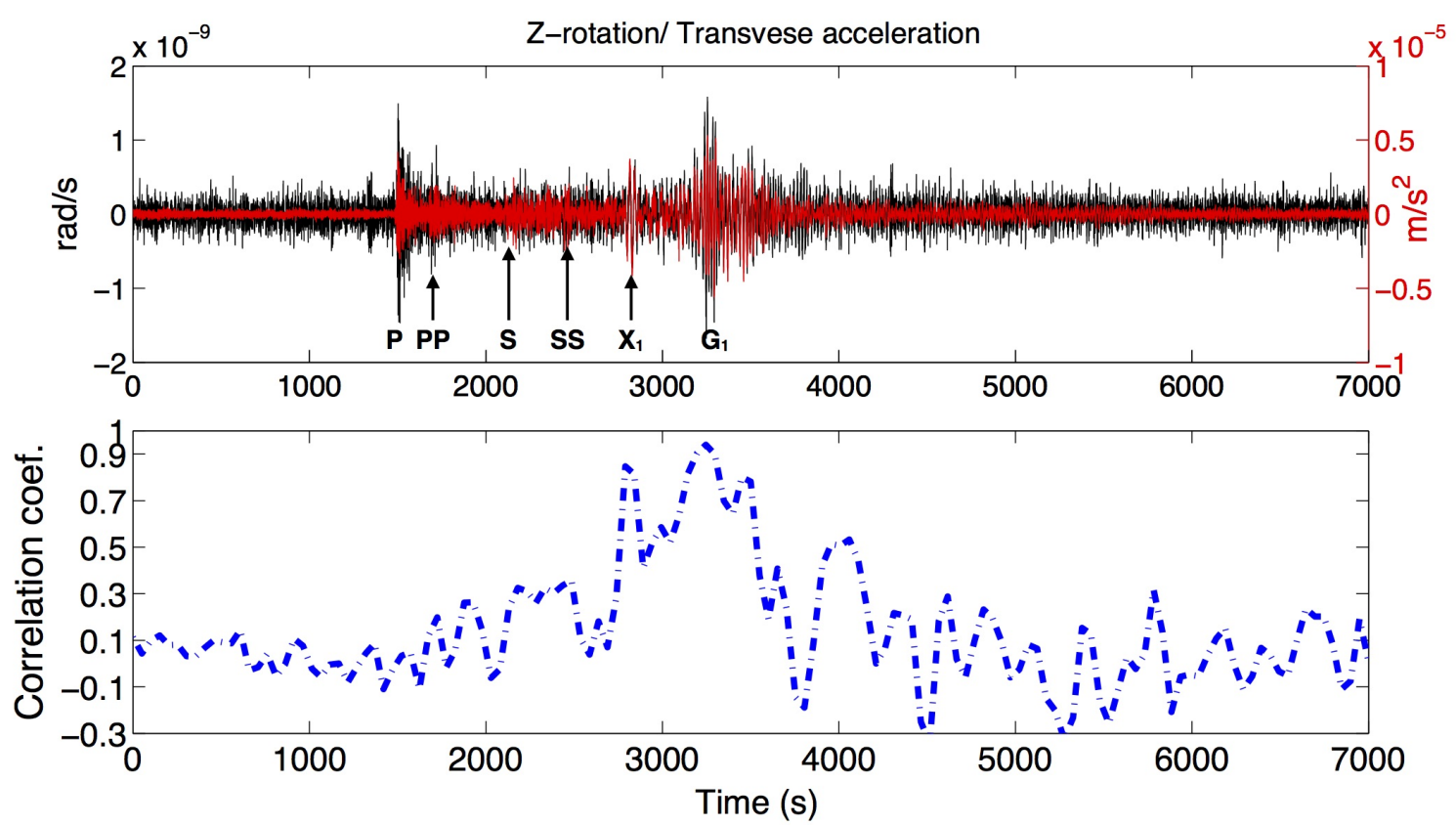

Figure 4: (top) Ground rotation and transverse acceleration time histories (black and red lines, respectively), time zero is at 12:40:00 UTC. (bottom) ZLCC between the above traces.

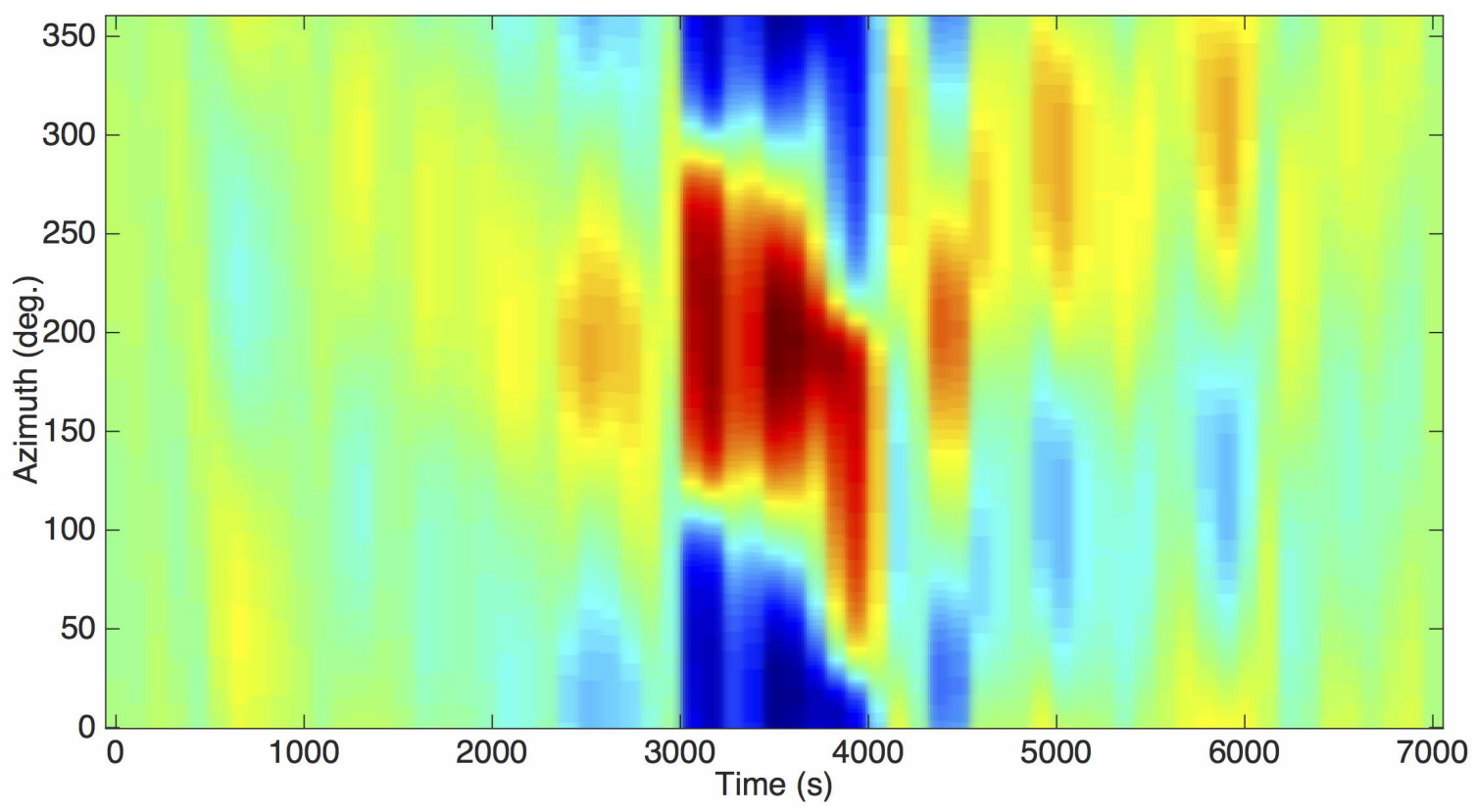

Figure 5: Zero-lag normalized correlation coefficient (defined between -1 (blue) and 1 (red)) between rotation rate and transverse acceleration, varying the unknown transverse direction from $0^{\circ}$ to $360^{\circ}$. The theoretical propagation azimuth is $202^{\circ}$. 


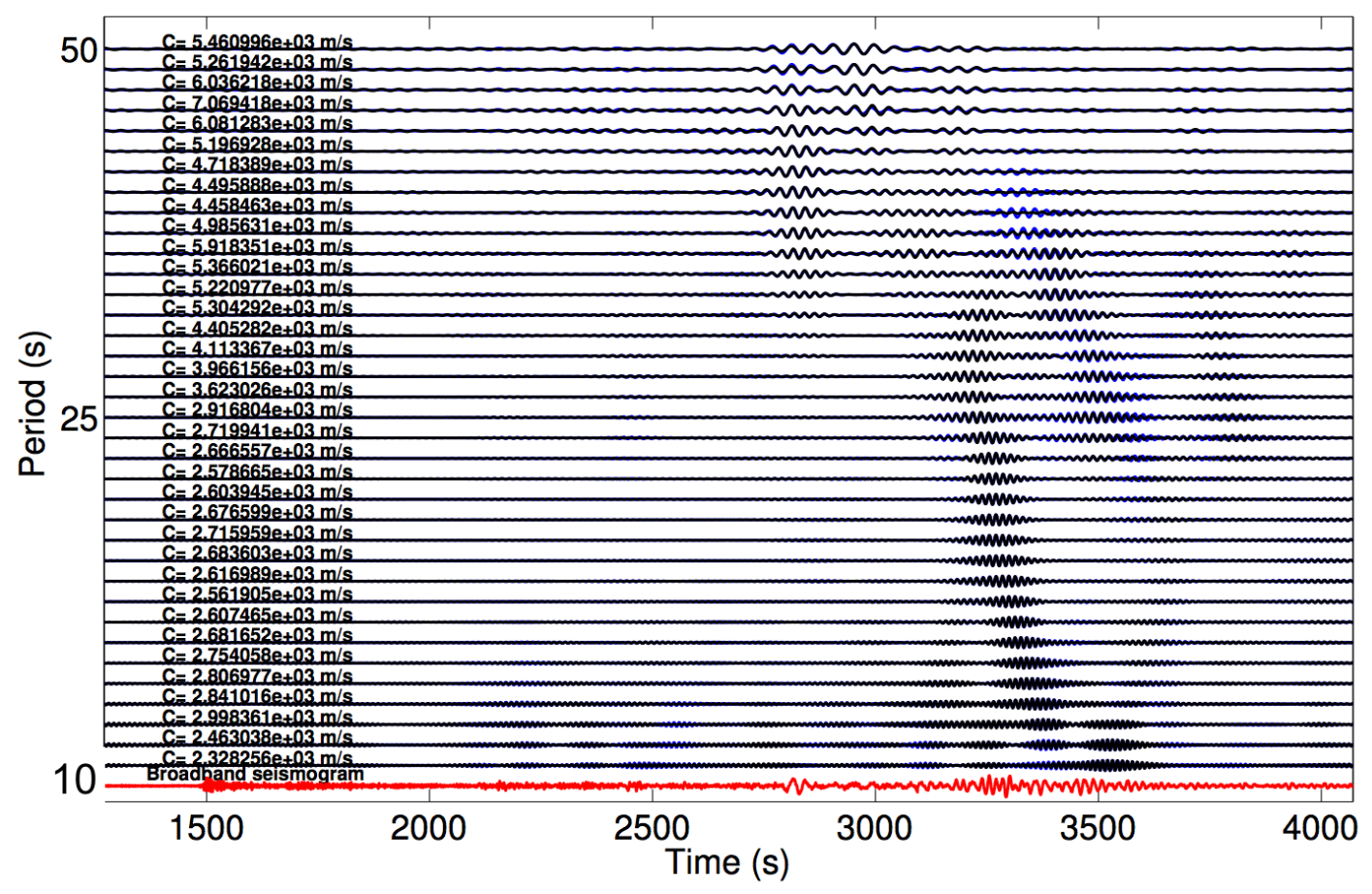

Figure 6: Superposition of transverse acceleration (black) and rotation rate (blue) and determination of phase velocities as a function of dominant period after narrow-band filtering. Each trace has been individually normalised. Phase velocities reported at the left side of the plot are obtained from the amplitude ratio of the envelope's maxima (see text for details)

order to monitor the possible slow ground tilt related to either local or wide scale (solid earth tides) effects.

\section{METHOD}

RLGs essentially consist of two laser beams counter-propagating within a closed-loop optical cavity (ring). If the cavity undergoes a rotation with respect to an inertial reference frame, then the optical path of the two beams changes, inducing a slight frequency shift between the clockwise and anti-clockwise propagating waves (Sagnac effect). This tiny frequency separation produces a characteristic beat note once the two beams are mixed outside the ring. The beat frequency $f$, also called the Sagnac frequency, is related to the rotation rate around the normal vector $n$ to the surface enclosed by the ring (see Fig. 2) through the equation:

$$
\Omega=\frac{\lambda_{\mathrm{He}: N e}}{L \cos \theta} f
$$

where $\lambda_{\mathrm{He}: \mathrm{Ne}}$ is the wavelength of the $\mathrm{He}: \mathrm{Ne}$ laser $(632 \mathrm{~nm}), L$ is the square side length of the optical cavity and $\theta$ is the angle between the versor $\hat{n}$ and $\vec{\Omega}$. We know from theory (Aki and Richards (2009)) that rotations can be retrieved from ground displacement as the curl of the wave-field.

$$
\vec{\Omega}=\frac{1}{2}(\nabla \times \vec{u})
$$

For example, the displacement caused by a Love wave traveling as a plane wave along the $x$-direction is expressed through the equation:

$$
u_{y}=A e^{i \omega\left(x / C_{L}-t\right)}
$$

By applying eq. 3 to eq. 2 we obtain the relationship:

$$
\Omega_{z}=\frac{\ddot{u}_{y}}{2 c_{L}}
$$


which provides a direct estimation of the phase velocity $C_{L}$ from a single-site measurement. From this latter formulation it is also evident that the sensing of ground rotations over the seismic frequency band requires high sensitivity: considering the range for shear wave velocities within the Earth, ground rotations are expected to be two to three orders of magnitude smaller than the associated translational movements. For this reason a very sensitive and completely decoupled from translations device is required, and at present large RLGs are the best candidates.

\section{FIRST RESUlts}

During the longest run of continuous data acquisition from June 11 to June 19, 2015 our instruments recorded an $\mathrm{M}=7$ earthquake occurred on June 17, 2015 12:51:32 (UTC), with epicenter at sea along the Southern Mid Atlantic Ridge. Though the recordings exhibit a poor signal-to-noise-ratio (SNR), their quality is sufficient to perform some analysis of seismological interest. The following processing steps have been performed. The N-S and E-W ground displacement traces are rotated with steps of $1^{\circ}$ over the $0^{\circ}, 360^{\circ}$ angular range. For each rotation step, the zerolag-cross-correlation (ZLCC) between the rotational signal and transverse accelerations is calculated. The maximum correlation is found at a rotation angle of $198^{\circ} \mathrm{N}$, which compares well with the $202^{\circ}$ $\mathrm{N}$ theoretical azimuth derived from epicenter and station coordinates. Such small discrepancy between the observed and theoretical azimuthal values is likely related to possible seismometer misorientation and deviation of surface wave trajectories from the great circle path as a consequence of lateral velocity heterogeneities.The ZLCC between translational and rotational traces is calculated using a $200 \mathrm{sec}-$ onds long window, sliding with $50 \%$ overlap. The Love-wave arrival is marked by a clear correlation peak (see Fig.4). Ground rotations and Transverse accelerations (respectively blue and black lines in Fig. 6) are band-pass filtered over narrow frequency bands using a FIR filter with 1-s bandwidth spanning the $1 \mathrm{~s}-50 \mathrm{~s}$ period range. In the frequency bands where ZLCC is above a threshold of 0.7 , the amplitude ratio between the maxima of the rotational and translational envelopes (evaluated via Hilbert transform) provides a direct measure of phase velocity for that particular period (see Fig. 6).

\section{Conclusions}

In this paper we presented the preliminary results from the operation of GINGERino, a Ring Laser Gyroscope co-located with a broad-band seismometer within the INFN's Gran Sasso laboratories. Our data constitutes the very first underground observations of earthquakegenerated rotational motions. The source backazimuth inferred from the directional analysis is in good agreement with the theoretical one, suggesting that the joint RLG - seismometer deployment can provide precise, singlesite estimation of the propagation direction of seismic wave-fields. For those time intervals in which the translational and rotational signals are significantly correlated, we also obtained estimates of Love-wave phase velocities, which span the $3800 \mathrm{~m} / \mathrm{s}-4500 \mathrm{~m} / \mathrm{s}$ range over the 10s-50s period interval. Such velocities are in good agreement with those expected for Love waves propagating in the PREM Earth's model (Dziewonski and Anderson (1981)). GINGERino is presently running in a preliminary test mode: current efforts are aimed at optimizing the experimental settings in order to increase the sensitivity and to achieve continuous acquisition. Such improvements will allow extending the seismological analyses to a ranges of magnitude larger than those considered until now (Belfi et al. (2012c)). The simultaneous measurement of broad-band ground translation and rotation will thus permit the definition of the dispersive properties of Love waves over a broad frequency range, from which a local shear-wave velocity profile can be inferred with resolutions on the order of $100 \mathrm{~m}$ and penetration depths up to several tens of kilometers. Sensibility improvements will also permit studying the partition of elas- 
tic energy in the microseism wavefield (e.g., Tanimoto et al. (2015)), whose main spectral peaks at the test site (3s and 10s) are at present only a factor five below our noise floor.

\section{REFERENCES}

Aki, K. and Richards, P. G. (2009). Quantitative Seismology. University Science Books.

Belfi, J., Beverini, N., Bosi, F., Carelli, G., Di Virgilio, A., Kolker, D., Maccioni, E., Ortolan, A., Passaquieti, R., and Stefani, F. (2012a). Performance of "G-pisa" ring laser gyro at the VIRGO site. Journal of Seismology, 16(4):757766.

Belfi, J., Beverini, N., Bosi, F., Carelli, G., Di Virgilio, A., Maccioni, E., Ortolan, A., and Stefani, F. (2012b). A $1.82 \mathrm{~m} 2$ ring laser gyroscope for nano-rotational motion sensing. Applied Physics B, 106(2):271-281.

Belfi, J., Beverini, N., Carelli, G., Di Virgilio, A., Maccioni, E., Saccorotti, G., Stefani, F., and Velikoseltsev, A. (2012c). Horizontal rotation signals detected by "G-Pisa" ring laser for the $\mathrm{m} \mathrm{w}=9.0$, March 2011, Japan earthquake. Journal of Seismology, 16(4):767-776.

Brokešová, J. and Málek, J. (2010). New portable sensor system for rotational seismic motion measurements. Review of Scientific Instruments, 81(8):084501.

Dziewonski, A. M. and Anderson, D. L. (1981). Preliminary reference earth model. Physics of the Earth and Planetary Interiors, 25(4):297 356.

Igel, H., Schreiber, U., Flaws, A., Schuberth, B., Velikoseltsev, A., and Cochard, A. (2005). Rotational motions induced by the M 8.1 Tokachi-Oki earthquake, September 25, 2003. Geophysical research letters, 32(8).

Kaláb, Z., Knejzlík, J., and Lednická, M. (2013). Application of newly developed rotational sensor for monitoring of mining induced seismic events in the Karvina region. Acta Geodyn Geomater, 10(2):170.
Pancha, A., Webb, T., Stedman, G., McLeod, D., and Schreiber, K. (2000). Ring laser detection of rotations from teleseismic waves. Geophysical Research Letters, 27(21):3553-3556.

Schreiber, K., Velikoseltsev, A., Rothacher, M., Klügel, T., Stedman, G., and Wiltshire, D. (2004). Direct measurement of diurnal polar motion by ring laser gyroscopes. Journal of Geophysical Research: Solid Earth (1978-2012), 109(B6).

Schreiber, K. U., Stedman, G. E., Igel, H., and Flaws, A. (2006a). Ring laser gyroscopes as rotation sensors for seismic wave studies. In Earthquake Source Asymmetry, Structural Media and Rotation Effects, pages 377390. Springer.

Schreiber, U., Igel, H., Cochard, A., Velikoseltsev, A., Flaws, A., Schuberth, B., Drewitz, W., and Müller, F. (2006b). The GEOsensor project: rotations, a new observable for seismology. In Observation of the Earth System from Space, pages 427-443. Springer.

Simonelli, A. (2014). Il giroscopio laser come sensore di rotazioni: simulazioni e attività sperimentale. Master's thesis, University of Pisa, https://etd.adm.unipi.it/theses/ available/etd-06202014-163524/.

Tanimoto, T., Hadziioannou, C., Igel, H., Wasserman, J., Schreiber, U., and Gebauer, A. (2015). Estimate of rayleigh-to-love wave ratio in the secondary microseism by colocated ring laser and seismograph. Geophysical Research Letters, 42(8):2650-2655. 